\title{
Opioid-Induced Constipation in Advanced Cancer Patients
}

Nabil ALMouaalamy 1, 2, 3

1. Oncology Department/Palliative Care, Princess Noorah Oncology Center, King Abdulaziz Medical City, Ministry of National Guard-Health Affairs, Jeddah, SAU 2. Research, King Abdullah International Medical Research Center, Jeddah, SAU 3. College of Medicine, King Saud Bin Abdulaziz University for Health Sciences, Jeddah, SAU

Corresponding author: Nabil ALMouaalamy, almoalami@yahoo.com

\begin{abstract}
The present study discusses opioid-induced constipation (OIC) in advanced cancer patients, focusing on the OIC definition, pathophysiology, and treatment. OIC is any change from baseline defecation patterns and bowel habits that developed after starting opioid therapy. The condition is characterized by bowel frequency reduction, worsening or development of straining, a sensation of incomplete defecation, or distress associated with bowel habits. OIC is common in advanced cancer patients, with a prevalence of approximately $51 \%-87 \%$ in patients taking opioids for pain management. Patients are likely to experience severe distress, work productivity reduction, poor quality of life, and increased healthcare utilization. OIC has a complex pathophysiology that involves propulsive and peristalsis impairment, intestinal mucosal secretion inhibition, intestinal fluid absorption enhancement, and anal sphincters function impairment. The Rome III criteria are used to assess and diagnose clinical OIC and can also be diagnosed through the Patient Assessment of Constipation (PAC) measures, including the symptom survey (PAC-SYM) and quality of life survey (PAC-QOL). Non-pharmacological treatment of OIC involves lifestyle habits and dietary adjustments, although these interventions might be insufficient to manage the condition. Pharmacological treatments involve the use of traditional laxatives and newer agents like peripherally acting mu-opioid receptor agonists (PAMORAs), including naldemedine, naloxegol, and methylnaltrexone. More novel treatments for OIC that target the pathophysiology are still needed and should be studied carefully for safety and efficacy.
\end{abstract}

Review began 04/06/2021 Review ended 04/06/2021 Published 04/09/2021

\section{() Copyright 2021}

ALMouaalamy. This is an open access article distributed under the terms of the Creative Commons Attribution License CC-BY 4.0., which permits unrestricted use, distribution, and reproduction in any medium, provided the original author and source are credited.
Categories: Family/General Practice, Internal Medicine, Oncology

Keywords: opioids, cancer, constipation

\section{Introduction And Background}

Pain is a serious concern for people who are diagnosed with different forms of cancer. Chiefly, pain prevalence rates are $39.3 \%$ following curative therapy, 66.4\%-80\% in advanced cancer, and 55\% during anticancer therapy [1]. There has been increased attention on pain in cancer patients because it affects the quality of life and is associated with many psychosocial responses. As pain is highly prevalent among cancer patients, pain alleviation is a critical treatment goal. The American Society of Clinical Oncology recommends the use of opioids to manage pain in selected cancer patients who are unresponsive to conservative pain management approaches and continue to experience functional impairment or distress [2]. Opioids are recommended for various types of cancer pains, such as neuropathic, visceral, and somatic pain, because of ease of titration, efficacy, and favorable safety profile [3].

Even though opioids are effective, they are linked to various adverse events, such as opioid-induced constipation (OIC), abdominal discomfort, oesophageal reflux, dry mouth, nausea, and vomiting [4]. Most adverse events, such as vomiting and nausea, disappear after few days, but OIC can persist throughout the opioid treatment period. Opioids prescription should incorporate necessary precautions to prevent adverse events, abuse, and addiction. OIC has a substantial effect on the quality of life for cancer patients on opioid treatment. Cancer patients tend to associate constipation with severe distress. Moreover, patients are likely to report work productivity reduction, low quality of life, and healthcare utilization increase. Cancer patients tend to discontinue or avoid opioid therapy because of OIC, which might cause them to sacrifice effective pain control to prevent constipation [3]. Therefore, OIC is emerging as a key aspect in cancer patients who use opioids for pain management. This paper discusses OIC, its pathology, and treatment options.

\section{Review \\ Definitions}

OIC is the most prevalent form of opioid-induced bowel disorder (OIBD). Opioid action on the gastrointestinal (GI) tract and central nervous system (CNS) or the unintended consequences of opioid therapy on the GI tract are collectively referred to as OIBD [5]. OIBD develops when opioids disrupt normal GI function by binding to opioid receptors [6]. OIC is defined by Camilleri M et al. as any change from baseline defecation patterns and bowel behaviors that developed after starting opioid therapy. This change 
is characterized by one of the following: 1) bowel frequency reduction, 2) worsening or development of straining, 3) a sensation of incomplete defecation, and 4) harder stool consistency [7]. Any of these changes can be a symptom of OIC if they develop upon opioid therapy initiation. A patient might also experience fecal impaction characterized by overflow incontinence, whereas other patients can experience symptoms consistent with overlapping OIBD such as bloating, nausea, and reflux [5].

OIC can be frequent in advanced cancer patients using opioid therapy to manage pain. There is no consensus on the actual OIC frequency among advanced cancer patients. Lacy et al. indicate that OIC prevalence is about $41 \%$ in those with chronic noncancer pain placed under opioid therapy [5]. Among cancer patients using opioids for pain control, the prevalence of constipation is almost 94\% [5]. In another study, Farmer et al. observe that OIC happens in $51 \%$ - $87 \%$ of cancer patients under opioid therapy and $41 \%$ $57 \%$ patients taking the therapy for chronic noncancer pain [8]. Even though OIC is a prevalent reason for constipation, other factors might influence constipation occurrence or worsen OIC symptoms in cancer patients. The differential diagnosis of OIC is important to determine the specific cause of constipation and offer effective treatment. Another important definition is laxative-refractory OIC, which is defined as inadequate laxative response with severe symptoms of constipation (bowel function index (BFI) ₹30), despite the scheduled use of two laxatives from two or more laxative classes for a minimum of four days within a two-week period [9].

\section{Pathophysiology}

OIC develops due to propulsive and peristalsis impairment, intestinal mucosal secretion inhibition, intestinal fluid absorption enhancement, and anal sphincters impairment. The disturbances in normal function or impairment occur because of opioid receptors' activation within the GI by exogenous opioids [6].

\section{Opioid-induced gut dysmotility}

OIC results from opioid-mediated activity on receptors in the GI and even in the CNS [3]. The primary opioid receptors are $\mu-, \mathrm{K}-$, and $\delta$-, and these receptors are dispersed across the GI tract [8]. The distribution of opioid receptors varies significantly based on the layer and specific region of the gut. High levels of $\mu$ - and $\mathrm{k}$ are found within the proximal colon and stomach [8]. On the other hand, $\mu$-receptors are mainly dispersed throughout the GI tract and largely localized with submucosal neurons and myenteric and on immune cells within the lamina propria [6].

Opioid receptors and opioid peptides are expressed by different intestinal muscle cells and enteric neurons. Upon release from neurons, opioid peptides serve as transmitters in the enteric modulation of peristalsis and propulsive motility. Chiefly, the impact of this mechanism is twofold. Firstly, it attenuates the normal peristalsis and propulsion. Secondly, it triggers non-propulsive motility patterns and tonic spasms in the colon and small intestines [3]. The cascade impact is a gastric-emptying delay, abdominal cramping, deceleration of intestinal transit, and eventually constipation.

\section{Opioids impact on the mucosal secretion}

About nine to 10 liters of fluid are secreted from the GI tract daily [8]. Opioids have a major impact on the absorptive and secretory GI's function via several mechanisms. Opioid receptor activation within the GI tract inhibits electrolyte and submucosal water secretion [3]. Also, opioid receptor activation raises fluid absorption from lumina space and retention. Treatment with opioids increases sympathetic tone, which decreases secretions into the lumen [3]. Ultimately, these effects increase the absorption of fluid from the lumen, and stools become hard and dry.

\section{Opioids impact on GI sphincters}

Opioids have a profound impact on defecation reflexes [3]. A human's GI tract has about six functionally or anatomically characterized sphincters, including the lower and upper esophageal sphincters, sphincter of Oddi, pylorus, anal sphincters, and ileocaecal valve [8]. Opioids can modulate the functioning of all these sphincters, which impact the GI tract's integrity. The anal sphincters play an important role in the OIC's pathology. Opioid-induced impairment of anorectal function is marked by an increase in internal anal sphincter contraction. The increased internal anal sphincter contraction leads to straining, hemorrhoids, and a feeling of incomplete defecation [8]. In particular, anal sphincter impairment decreases anal and rectal sensitivity to the rectal vault and creates a sense of anal blockage, common among patients with OIC [3]. A sense of incomplete defecation, straining, and hemorrhoids together can result in severe challenges with defecation, and colonic perforation might happen in the worst scenario. For example, loperamide tends to increase the internal sphincter tone, and about a third of patients placed under opioids treatment report a feeling of anal blockage even after laxative treatment [8]. These findings have been reproduced in recent studies. Poulsen et al. found that oxycodone impairs anal sphincter relaxation, and slow-release naloxone can reverse this effect [10].

\section{Assessment and diagnosis of OIC}

Clinical OIC Assessment and Diagnosis 


\section{Cureus}

Patients who are being treated with opioids might present with constipation because of several factors that might not be identified on initial assessment [8]. Opioid treatment might not be the primary cause but may worsen the symptoms. Clinicians should take an in-depth patient history when assessing patients who present with constipation. A clinician should focus on the normal bowel habit and changes after opioid therapy introduction. Patients are likely to identify potential changes in bowel movement that occurred when opioid treatment was initiated. Comprehensive drug history is required during the assessment to determine drugs that may be causing constipation.

The patient's health history and current medications might be insufficient to assess and diagnose OIC in cancer patients. Rome III criteria (Table 1) can be used for OIC diagnosis in clinical settings [5]. The Rome III criteria have proven effective in defining OIC in clinical practice and clinical trials [11]. Even though Rome III criteria have been created for functional constipation, they offer a standardized OIC definition. This assessment standard comprises straining at defecation, hard stools, anorectal obstruction or a feeling of incomplete defecation, reliance on manual aids to facilitate stools passage, and passing under three stools a week [11].

\section{Diagnostic Criteria for Opioid-Induced Constipation}

1. New, or worsening, symptoms of constipation when initiating, changing, or increasing opioid therapy that must include 2 or more of the following:

a. Straining during more than one-fourth $(25 \%)$ of defecations

b. Lumpy or hard stools (BSFS 1-2) more than one-fourth (25\%) of defecations

c. Sensation of incomplete evacuation more than one-fourth $(25 \%)$ of defecations

d. Sensation of anorectal obstruction/blockage more than one-fourth (25\%) of defecations

e. Manual maneuvers to facilitate more than one- fourth (25\%) of defecations (eg, digital evacuation, support of the pelvic floor)

f. Fewer than three spontaneous bowel movements per week

2. Loose stools are rarely present without the use of laxatives

TABLE 1: Rome III diagnostic criteria for opioid-induced constipation

Source [5]

Furthermore, digital rectal examinations are recommended in patients consulting for opioid-induced constipation. Digital rectal examinations are important for clinicians to exclude anorectal malignancy, minor anal pathologies, such as anal fissure and fecal impaction, which likely worsen symptoms [8]. Systematic bowel function review is recommended for patients starting opioids treatment and maintaining opioids treatment due to OIC prevalence.

Patient-Reported OIC Outcome Measures

Several scales are available to help in the diagnosis of OIC among cancer patients. Patient-reported scales for assessing OIC have been validated in patients with different cancers. The Bowel Function Index (BFI) has received a lot of attention because of its simplicity [3]. Importantly, the BFI is a survey administered by a clinician that consists of three questions to assess defecation ease, the sense of incomplete defecation, and the patient's personal judgment concerning constipation [3]. A patient scores the questions based on his or her personal experiences in the last seven days. The three questions are scored through a numeric scale ranging from $0-100$, and the mean score is treated as the final score. Zero (0) means no symptom while 100 means severe symptoms. The BFI is reliable and seems responsive to changes in OIC severity [3]. A 12-point change in the BFI scale is considered a clinically important constipation change. A consensus panel on OIC and other opioid-associated negative events recommends treatment for patients with a BFI score of $\geqslant 30$ [12]. The consensus panel advocates the BFI scale application because of its ease of use (See Table 2). 


\section{Cureus}

\begin{tabular}{|l|l|l|}
\hline Item & Question & Scale \\
\hline 1 & $\begin{array}{l}\text { During the last } 7 \text { days, how would you rate your ease of defecation on a scale from } 0 \\
\text { to } 100 ?\end{array}$ & $\begin{array}{l}0=\text { easy or no difficulty } 100= \\
\text { severe difficulty }\end{array}$ \\
\hline 2 & $\begin{array}{l}\text { During the last } 7 \text { days, how would you rate your feeling of incomplete bowel } \\
\text { evacuation on a scale from } 0 \text { to } 100 ?\end{array}$ & $0=$ not at all $100=$ very strong \\
\hline 3 & $\begin{array}{l}\text { During the last } 7 \text { days, how would you rate your constipation on a scale from } 0 \text { to } \\
100 ?\end{array}$ & $0=$ not at all $100=$ very strong \\
\hline $\begin{array}{l}\text { Total } \\
\text { score }\end{array}$ & & Mean of 3 scores \\
\hline
\end{tabular}

TABLE 2: Bowel function index

The Patient Assessment of Constipation (PAC) is widely used to assess OIC in cancer patients. Among the common PAC, measures include the "symptom survey (PAC-SYM) and quality of life survey (PAC-QOL)" [3]. The PAC-SYM questionnaire was created via psychometric assessment of patients with chronic constipation. This questionnaire is considered effective in evaluating the severity of symptoms in patients with OIC. The survey has 12 items divided into 3 symptom subscales, including rectal (3 items), stool (5 items), and abdominal (4 items) [13]. Items of this scale are scored through five-point Likert scales, ranging from 0-4. In this case, 0 shows the absence of symptoms, while 4 indicates very severe symptoms. The total score is divided by a number of questions to get an average total score, ranging from 0-4 [12]. A low average score shows a low symptom burden.

On the contrary, PAC-QOL consists of 28 items that are divided into psychological discomfort, physical discomfort, dissatisfaction, and concerns and worries [3]. These four domains are meant to assess the extent to which OIC symptoms are impacting a patient. Rumman et al. indicate that PAC is beneficial because it is comprehensive and assesses a patient's quality of life and OIC symptoms burden [3]. The patient-reported outcome measures have become important because of the increasing concern of OIC among cancer patients. Nevertheless, most patient-reported outcome measures are too complex for routine clinical practice.

\section{Treatment}

Non-Pharmacological Treatment

Dietary interventions might improve OIC symptoms and improve a patient's quality of life. According to Gregorian et al., adjustments in lifestyle habits and diet can substantially improve OIC and other forms of constipation [14]. A patient can improve constipation by consuming 25-30 $\mathrm{g}$ of soluble fiber daily, having a balanced diet, sufficient fluids, regular aerobic exercise, regular meal patterns, and avoiding fat, heavy meat, flatulent foods, and insoluble fiber [5]. Rumman et al. acknowledge that non-pharmacological treatments alone might be insufficient to OIC management but act as pharmacology treatment adjuncts [3]. Patient education concerning activity and fluid intake should be emphasized where possible to help patients prevent OIC.

\section{Pharmacological Treatment}

The American Gastroenterological Association (AGA) recommended the use of laxatives as the first-line in patients with OIC [9]. Several studies show that stimulant laxatives (senna, bisacodyl, and picosulphate) and osmotic laxatives (polyethylene glycol) should be the first choice in OIC patients [15-16]. These medications can be used as first-line options for OIC management (see Table 3). However, laxatives have several adverse side effects such as gas, fullness/bloating, and defecation urgency [8]. 


\section{Cureus}

\begin{tabular}{|c|c|c|c|}
\hline Class/type & Examples & Mechanism of action & Role in cancer patients \\
\hline \multicolumn{4}{|l|}{ Traditional laxatives } \\
\hline Bulking Agents & $\begin{array}{l}\text { Soluble (e.g., } \\
\text { psyllium, pectin) } \\
\text { Insoluble } \\
\text { (methylcellulose) }\end{array}$ & $\begin{array}{l}\text { Induce a stretch reflex in the intestinal wall, which } \\
\text { increases the colonic motility, water absorption, and } \\
\text { bacterial proliferation in the colon, leading to softening } \\
\text { the stool and a smoother process. }\end{array}$ & $\begin{array}{l}\text { Bulk laxatives are not effective } \\
\text { for already-constipated cancer } \\
\text { patients. }\end{array}$ \\
\hline Osmotic & $\begin{array}{l}\text { PEG, lactulose, } \\
\text { magnesium } \\
\text { citrate, } \\
\text { magnesium } \\
\text { hydroxide }\end{array}$ & Draw water into intestine to hydrate and soften stool & $\begin{array}{l}\text { Yes, PEG is one of the first-line } \\
\text { recommendations }\end{array}$ \\
\hline Stimulant & $\begin{array}{l}\text { Bisacodyl, } \\
\text { sodium } \\
\text { picosulfate, } \\
\text { senna }\end{array}$ & $\begin{array}{l}\text { Irritate sensory nerve endings to stımulate colonic } \\
\text { motility and reduce colonic water absorption }\end{array}$ & Yes, first-line recommendation \\
\hline $\begin{array}{l}\text { Detergent/surfactant } \\
\text { stool softeners }\end{array}$ & Docusate & $\begin{array}{l}\text { Allow water and lipids to penetrate the stool to hydrate } \\
\text { and soften fecal material }\end{array}$ & $\begin{array}{l}\text { Patients must have enough fluid } \\
\text { intake with these agents and it }\end{array}$ \\
\hline Lubricant & Mineral oil & Lubricate the lining of the gut to facilitate defecation & have to be added. \\
\hline \multirow{3}{*}{ PAMORAS } & Naldemedine & \multirow{3}{*}{$\begin{array}{l}\text { Block } \mu \text {-opioid receptors in the gut, thereby effectively } \\
\text { restoring the function of the enteric nervous system }\end{array}$} & Yes \\
\hline & Naloxegol & & Yes \\
\hline & Methylnaltrexone & & Yes \\
\hline $\begin{array}{l}\text { Intestinal } \\
\text { secretagogues }\end{array}$ & Lubiprostone & $\begin{array}{l}\text { Act on chloride channels or guanylate cyclase receptors } \\
\text { in enterocytes to stimulate fluid secretion into the } \\
\text { intestinal lumen }\end{array}$ & $\begin{array}{l}\text { No, AGA. No recommendation } \\
\text { due to evidence gap. }\end{array}$ \\
\hline $\begin{array}{l}\text { Selective 5-HT } \\
\text { agonists }\end{array}$ & Prucalopride & $\begin{array}{l}\text { Activate } 5-\mathrm{HT} 4 \text { receptor, leading to increased colonic } \\
\text { motility and accelerated transit }\end{array}$ & $\begin{array}{l}\text { No, AGA. No recommendation, } \\
\text { due to evidence gap. }\end{array}$ \\
\hline
\end{tabular}

\section{TABLE 3: Different classes of agents for opioid-induced constipation and mechanisms of action}

Note. Adapted from [9]

AGA: American Gastroenterological Association; PEG: polyethylene glycol; PAMORA: peripherally acting mu-opioid receptor agonist

Also, opioid switching or using another opioid that is less constipating is a solution [17]. For example, transdermal opioids like fentanyl may cause less constipation than morphine in oral or parenteral preparations [18]. Also, the use of combination opioid agonist/antagonist agents (eg, oxycodone + naloxone) may decrease the risk of constipation [19]. The Food and Drug Administration (FDA)-approved prescription products for OIC are lubiprostone, naloxegol, methylnaltrexone, and naldemedine [14]. It is recommended to use the AGA OIC clinical decision tool when trying to approach a patient with OIC (Figure 1) [9]. 


\section{Opioid-Induced Constipation (OIC) Clinical Decision Support Tool}

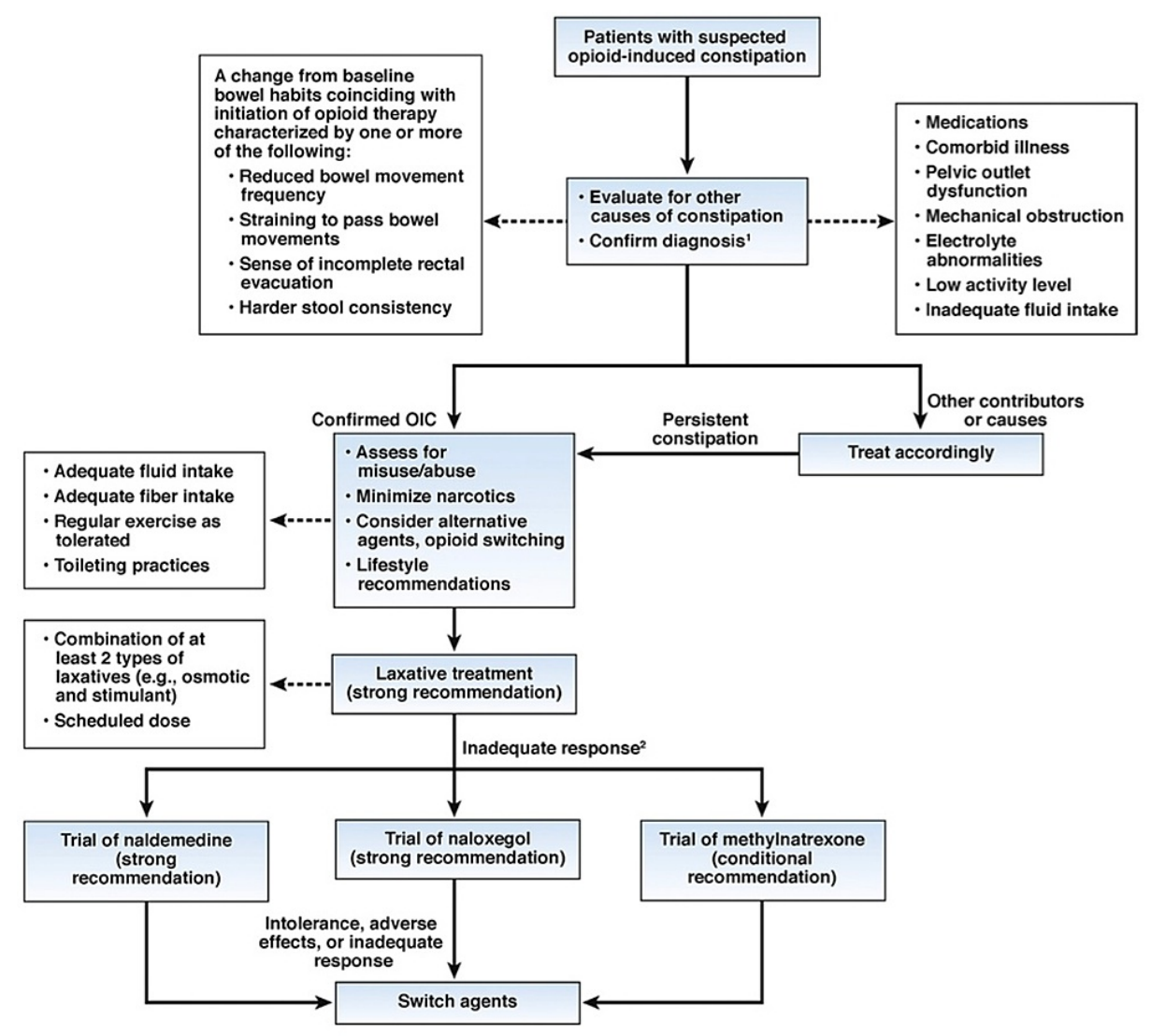

'OIC definition from Camilleri et al. Neurogastroenterol Motil. 2014

${ }^{2}$ Laxative refractory OIC defined as persistent constipation (e.g. Bowel Function Index score $\geq 30$ ) despite scheduled use of at least 2 classes of laxatives for at least 2 weeks

FIGURE 1: AGA OIC clinical decision tool

Source [9]

AGA: American Gastroenterological Association; OIC: opioid-induced constipation

Lubiprostone

Lubiprostone is a Cl- channel activator that increases the secretion of intestinal fluid and enhances fecal transit within the GI tract [20]. This product also alters gut mucus composition and flora to produce an antiinflammatory luminal milieu [3]. The recommended dosage of this product is $24 \mathrm{mcg}$ twice each day and should be taken with water and food [14]. Patients with irritable bowel syndrome can take 8 mcg capsules of lubiprostone. Due to dosage variations, it is important for pharmacists to ensure that they prescribe the correct dose. Lubiprostone has been found to improve bowel movement and stool consistency significantly as compared to placebo, although it does not relieve abdominal pain [21].

Among the most prevalent side effects of using lubiprostone for OIC treatment are diarrhea and nausea [20]. Furthermore, hypotension, dyspnea, and syncope are likely to occur after the completion of the first dose. Most of these effects disappear on their own but might reoccur in subsequent doses. Dose modification is not needed for patients with renal impairment, but adjustments may be essential for patients with hepatic impairment. The likelihood of drug-drug interactions is minimal, as CYP450 isoenzymes do not modulate lubiprostone metabolism [14]. Lubiprostone can be tolerated for about 13 months, meaning it is effective for long-term use.

Peripherally Acting Mu-Opioid Receptor Agonists (PAMORAs)

Methylnaltrexone, naldemedine, and naloxegol are a class of PAMORAs since they block peripheral mu- 
opioid receptors and do not inhibit opiates' analgesic effects on the mu receptor in the CNS [14]. Nevertheless, patients taking PAMORAs have a risk of opioids withdrawal and need to be monitored for anxiety, rhinorrhea, hyperhidrosis, and chills. More importantly, opioid antagonists like naltrexone and naloxone should not be used together with PAMORAs due to the risk of addiction and withdrawal [14]. A patient should only be prescribed PAMORAs if they have taken opioids for four weeks and laxatives need to be discontinued [14]. Each PAMORA has distinct attributes despite being in the same class.

Methylnaltrexone

Methylnaltrexone is available in subcutaneous (SC) injection and oral formulations. The N-methyl group in this drug inhibits the capacity to cross the blood-brain barrier because of its low lipid solubility and polarity [8]. SC-injection formulation was approved for OIC treatment in advanced cancer patients who failed to respond sufficiently to laxatives. The recommended doses of SC injection are $12 \mathrm{mg}$ for $114 \mathrm{~kg}$ and $8 \mathrm{mg}$ for $62 \mathrm{~kg}[8]$.

The recommended dose is $450 \mathrm{mg}$ once per day for oral methylnaltrexone and should be taken 30 min before the first meal [14]. Siemens and Becker designed a meta-analysis study to identify the safety and effectiveness of methylnaltrexone in OIC patients [22]. Based on the findings, patients being treated with methylnaltrexone had rescue-free bowel movement only four hours after the initial dose, higher stool regularity, and required less time to laxation than placebo [22]. These patients also had greater levels of satisfaction, better outcomes, and minimal adverse events.

Naloxegol

Naloxegol is approved for OIC treatment in patients with chronic pain related to cancer and non-cancer pain. Although the recommended dose is $25 \mathrm{mg}$ per day, it can be tapered to $12.5 \mathrm{mg}$ if not well-tolerated [14]. Oral intake is recommended, but this product might be crushed and administered through a nasogastric tube where necessary. Just like methylnaltrexone, naloxegol should be taken on an empty stomach. A patient can take this medication one hour before eating or two hours after eating [14]. Patients taking naloxegol should avoid certain products such as grapefruits and juice. The side effects of taking naloxegol are flatulence, nausea, vomiting, abdominal pain, headache, diarrhea, and arthralgia [14]. Double-blind studies meant to study the safety and efficacy of naloxegol have found that response rates are higher in patients treated with naloxegol than placebo without decreasing opioid-mediated analgesia [23]. They have concluded that the treatment is effective for OIC treatment. A newly published observational study for using naloxegol in 126 cancer patients found that it did improve quality of life and relieve constipation, which lasted for the whole follow-up period, which was one year [24].

Naldemedine

As naldemedine is structurally comparable to naltrexone, it has been categorized as a Schedule II controlled product. A patient can take a dose of $0.2 \mathrm{mg}$ per day with or without an empty stomach [14]. Even though renal adjustment is unnecessary, patients with hepatic impairment should avoid this product. Patients are likely to experience a range of side effects, including gastroenteritis, abdominal pain, nausea, and diarrhea. P-glycoprotein inhibitors and strong and moderate CYP3A4 inhibitors might raise naldemedine concentrations [14]. Patients should be monitored for adverse reactions if they are taking these medications. A 52-week study established the long-term safety of naldemedine [25]. Naldemedine was approved for OIC treatment based on the outcomes of this long-term study. Multiple studies show that naldemedine is effective and safe to use in cancer patients as well [26-27].

\section{Conclusions}

OIC has transitioned from a poorly understood disorder to a well-defined clinical disorder. Major milestones have been achieved in defining OIC, elaborating its complex pathology and effect on the quality of life. Opioid therapy affects GI tract function, leading to changes in defecation patterns and bowel habits. Cancer patients taking opioids to treat OIC are likely to experience distress and poor quality of life. Existing and novel treatments for OIC have been studied comprehensively and validated. Stimulant and osmotic laxatives are the primary treatments for OIC. Novel treatments have been approved in the last few years, mainly for patients who fail to respond to conventional laxative treatment. PAMORAs are among the recently approved therapeutics and include methylnaltrexone, naloxegol, and naldemedine. Long-term safety and efficacy studies should be done carefully to ensure only effective therapeutics are approved. It is recommended to use the AGA OIC clinical decision tool when trying to approach a patient with OIC.

\section{Additional Information}

\section{Disclosures}

Conflicts of interest: In compliance with the ICMJE uniform disclosure form, all authors declare the following: Payment/services info: All authors have declared that no financial support was received from any organization for the submitted work. Financial relationships: All authors have declared that they have 
no financial relationships at present or within the previous three years with any organizations that might have an interest in the submitted work. Other relationships: All authors have declared that there are no other relationships or activities that could appear to have influenced the submitted work.

\section{References}

1. van den Beuken-van Everdingen MH, Hochstenbach LM, Joosten EA, Tjan-Heijnen VC, Janssen DJ: Update on prevalence of pain in patients with cancer: systematic review and meta-analysis. J Pain Symptom Manage. 2016, 51:1070-1090.e9. 10.1016/j.jpainsymman.2015.12.340

2. Paice JA, Portenoy R, Lacchetti C, et al.: Management of chronic pain in survivors of adult cancers: American Society of Clinical Oncology Clinical Practice Guideline. J Clin Oncol. 2016, 34:3325-45. 10.1200/JCO.2016.68.5206

3. Rumman A, Gallinger ZR, Liu LWC: Opioid induced constipation in cancer patients: pathophysiology, diagnosis and treatment. Expert Review of Quality of Life in Cancer Care. 2016, 1:25-35. 10.1080/23809000.2016.1131595

4. Sarrió RG, Calsina-Berna A, García AG, Esparza-Miñana JM, Ferrer EF, Porta-Sales J: Delphi consensus on strategies in the management of opioid-induced constipation in cancer patients. BMC Palliat Care. 2021, 20:1. 10.1186/s12904-020-00693-Z

5. Mearin F, Lacy BE, Chang L, Chey WD, Lembo AJ, Simren M, Spiller R: Bowel disorders. Gastroenterology. 2016, 150:P1393-1407.E5. 10.1053/j.gastro.2016.02.031

6. Poulsen JL, Brock C, Olesen AE, Nilsson M, Drewes AM: Evolving paradigms in the treatment of opioidinduced bowel dysfunction. Therap Adv Gastroenterol. 2015, 8:360-72. 10.1177/1756283X15589526

7. Camilleri M, Drossman DA, Becker G, Webster LR, Davies AN, Mawe GM: Emerging treatments in neurogastroenterology: a multidisciplinary working group consensus statement on opioid-induced constipation. Neurogastroenterol Motil. 2014, 26:1386-95. 10.1111/nmo.12417

8. Farmer AD, Drewes AM, Chiarioni G, De Giorgio R, O'Brien T, Morlion B, Tack J: Pathophysiology and management of opioid-induced constipation: European expert consensus statement. United European Gastroenterol J. 2019, 7:7-20. 10.1177/2050640618818305

9. Crockett SD, Greer KB, Heidelbaugh JJ, Falck-Ytter Y, Hanson BJ, Sultan S: American Gastroenterological Association Institute guideline on the medical management of opioid-induced constipation. Gastroenterology. 2019, 156:218-26. 10.1053/j.gastro.2018.07.016

10. Poulsen JL, Mark EB, Brock C, Frøkjær JB, Krogh K, Drewes AM: Colorectal transit and volume during treatment with prolonged-release oxycodone/naloxone versus oxycodone plus Macrogol 3350. J Neurogastroenterol Motil. 2018, 24:119-27. 10.5056/jnm17058

11. Drossman DA, Hasler WL: Rome IV-functional GI disorders: disorders of gut-brain interaction . Gastroenterology. 2016, 150:1257-61. 10.1053/j.gastro.2016.03.035

12. Argoff CE, Brennan MJ, Camilleri M, et al.: Consensus recommendations on initiating prescription therapies for opioid-induced constipation. Pain Med. 2015, 16:2324-37. 10.1111/pme.12937

13. Yiannakou Y, Tack J, Piessevaux H, et al.: The PAC-SYM questionnaire for chronic constipation: defining the minimal important difference. Aliment Pharmacol Ther. 2017, 46:1103-11. 10.1111/apt.14349

14. Gregorian T, Lewis J, Tsu L: Opioid-induced constipation: clinical guidance and approved therapies . Gastroenterology. 2017, 42:15-19.

15. Chamberlain BH, Cross K, Winston JL, Thomas J, Wang W, Su C, Israel RJ: Methylnaltrexone treatment of opioid-induced constipation in patients with advanced illness. J Pain Symptom Manage. 2009, 38:683-90. 10.1016/j.jpainsymman.2009.02.234

16. Wirz S, Nadstawek J, Elsen C, Junker U, Wartenberg HC: Laxative management in ambulatory cancer patients on opioid therapy: a prospective, open-label investigation of polyethylene glycol, sodium picosulphate and lactulose. Eur J Cancer Care (Engl). 2012, 21:131-40. 10.1111/j.1365-2354.2011.01286.X

17. Caraceni A, Hanks G, Kaasa S, et al.: Use of opioid analgesics in the treatment of cancer pain: evidencebased recommendations from the EAPC. Lancet Oncol. 2012, 13:e58-68. 10.1016/S1470-2045(12)70040-2

18. Allan L, Richarz U, Simpson K, Slappendel R: Transdermal fentanyl versus sustained release oral morphine in strong-opioid naïve patients with chronic low back pain. Spine (Phila Pa 1976). 2005, 30:2484-90. 10.1097/01.brs.0000186860.23078.a8

19. Müller-Lissner S, Bassotti G, Coffin B, et al.: Opioid-induced constipation and bowel dysfunction: a clinical guideline. Pain Med. 2017, 18:1837-63. 10.1093/pm/pnw255

20. Wilson N, Schey R: Lubiprostone in constipation: clinical evidence and place in therapy . Ther Adv Chronic Dis. 2015, 6:40-5. 10.1177/2040622314567678

21. Jamal MM, Adams AB, Jansen JP, Webster LR: A randomized, placebo-controlled trial of lubiprostone for opioid-induced constipation in chronic noncancer pain. Am J Gastroenterol. 2015, 110:725-32. 10.1038/ajg.2015.106

22. Siemens W, Becker G: Methylnaltrexone for opioid-induced constipation: review and meta-analyses for objective plus subjective efficacy and safety outcomes. Ther Clin Risk Manag. 2016, 12:401-12. 10.2147/TCRM.S80749

23. Chey WD, Webster L, Sostek M, Lappalainen J, Barker PN, Tack J: Naloxegol for opioid-induced constipation in patients with noncancer pain. N Engl J Med. 2014, 370:2387-96. 10.1056/NEJMoa1310246

24. Cobo Dols M, Beato Zambrano C, Cabezón-Gutiérrez L, et al.: One-year efficacy and safety of naloxegol on symptoms and quality of life related to opioid-induced constipation in patients with cancer: KYONAL study. BMJ Support Palliat Care. 2021, [Epub ahead of print]:10.1136/bmjspcare-2020-002816

25. Webster LR, Nalamachu S, Morlion B, Reddy J, Baba Y, Yamada T, Arjona Ferreira JC: Long-term use of naldemedine in the treatment of opioid-induced constipation in patients with chronic noncancer pain: a randomized, double-blind, placebo-controlled phase 3 study. Pain. 2018, 159:987-94. 10.1097/j.pain.0000000000001174

26. Osaka I, Ishiki H, Yokota T, Tada Y, Sato H, Okamoto M, Satomi E: Safety and efficacy of naldemedine in cancer patients with opioid-induced constipation: a pooled, subgroup analysis of two randomised controlled 


\section{Cureus}

studies. ESMO Open. 2019, 4:e000527. 10.1136/esmoopen-2019-000527

27. Katakami N, Harada T, Murata T, et al.: Randomized phase III and extension studies of naldemedine in patients with opioid-induced constipation and cancer. J Clin Oncol. 2017, 35:3859-66.

10.1200/JCO.2017.73.0853 